Meta

Journal des traducteurs

Translators' Journal

\title{
Le rôle des chiasmes dans la naissance et la formation de la langue arabe
}

\section{André Roman}

Volume 55, numéro 1, mars 2010

Le parcours du sens : d'une langue à l'autre — Mélanges offerts à André Clas

The Way of Meaning: From a Language to Another - Collection of Articles Offered to André Clas

URI : https://id.erudit.org/iderudit/039613ar

DOI : https://doi.org/10.7202/039613ar

Aller au sommaire du numéro

Éditeur(s)

Les Presses de l'Université de Montréal

ISSN

0026-0452 (imprimé)

1492-1421 (numérique)

Découvrir la revue

Citer cet article

Roman, A. (2010). Le rôle des chiasmes dans la naissance et la formation de la langue arabe. Meta, 55(1), 198-207. https://doi.org/10.7202/039613ar
Résumé de l'article

Le système syllabique $\Sigma$, de l'arabe, qui comprend les seules syllabes \{Consonne-Voyelle\} et \{Consonne-Voyelle-Consonne\}, a déterminé l'impossibilité, dans le fonctionnement de la langue, de l'opposition d'une consonne à une voyelle. Ce chiasme est à l'origine de l'organisation ancienne des langues sémitiques, régissant, jusqu'à récemment, la langue arabe. Ainsi, la langue arabe a construit son système de nomination sur des arrangements de consonnes, les racines de ses unités de nomination, et elle a fait de ses voyelles désinentielles les signifiants de ses fonctionnels primitifs, ses cas. On relève, parmi d'autres, le chiasme organisant le verbe en deux paradigmes, achevé et non achevé. D'autres encore ont dans la langue l'apparence d'un ornement. Ces quelques cas exceptés, c'est, dans le discours, hors langue - la systématique des langues est une systématique fermée - que la rhétorique trouve la liberté qui lui est nécessaire.
Ce document est protégé par la loi sur le droit d'auteur. L'utilisation des services d’Érudit (y compris la reproduction) est assujettie à sa politique d'utilisation que vous pouvez consulter en ligne.

https://apropos.erudit.org/fr/usagers/politique-dutilisation/ 


\title{
Le rôle des chiasmes dans la naissance et la formation de la langue arabe
}

\author{
ANDRÉ ROMAN \\ Université Lumière - Lyon 2, Lyon, France \\ andre.roman@libertysurf.fr
}

\begin{abstract}
RÉSUMÉ
Le système syllabique $\Sigma$, de l'arabe, qui comprend les seules syllabes \{Consonne-Voyelle\} et Consonne-Voyelle-Consonne\}, a déterminé l'impossibilité, dans le fonctionnement de la langue, de l'opposition d'une consonne à une voyelle. Ce chiasme est à l'origine de l'organisation ancienne des langues sémitiques, régissant, jusqu'à récemment, la langue arabe. Ainsi, la langue arabe a construit son système de nomination sur des arrangements de consonnes, les racines de ses unités de nomination, et elle a fait de ses voyelles désinentielles les signifiants de ses fonctionnels primitifs, ses cas. On relève, parmi d'autres, le chiasme organisant le verbe en deux paradigmes, achevé et non achevé. D'autres encore ont dans la langue l'apparence d'un ornement. Ces quelques cas exceptés, c'est, dans le discours, hors langue - la systématique des langues est une systématique fermée - que la rhétorique trouve la liberté qui lui est nécessaire.
\end{abstract}

\begin{abstract}
The syllabic system, $\Sigma$, of the Arabic language is made up of no more than two patterns \{Consonant-Vowel\} and \{Consonant-Vowel-Consonant\}. Therefore two given strings of syllables CV and CVC cannot form a pair. There is, inevitably, a double opposition $\{\mathrm{C} v \mathrm{~s}$ $\mathrm{V}\}$. In that chiasmus lies the organization of all Semitic languages. In the Arabic language that chiastic organization has been maintained until recently. Therefore the Arabic language constructed its system of nomination on combinations of consonants that constitute the roots of its units of nomination. And it used the end vowels of those units as the signifiants of its primitive function words. Another example of a chiasmus is the chiasmus that distinguishes the perfective aspect of Arabic verbs from their imperfective aspect. Some chiasmi occurring in the language seem rhetorical, but such cases are rare. In fact, the systematic organization of the language is a closed one, while the discourse is open. And it is in the discourse that rhetoric finds the liberty that it requires.
\end{abstract}

\section{MOTS-CLÉS/KEYWORDS}

organisation binaire des langues, langues sémitiques, arabe, rhétorique, chiasme language binary organization, Semitic languages, Arabic, rhetoric, chiasmus

Le chiasme est une figure particulière de la répétition. Dans le discours, il peut embrasser plusieurs éléments dans des ordres complexes ${ }^{1}$. En langue, il embrasse deux seuls éléments, deux unités solidaires, $a$ et $b$, reprises dans un ordre inversé:

$\begin{array}{ll}\mathrm{a} & \mathrm{b} \\ \mathrm{b} & \mathrm{a}\end{array}$

Les langues se sont construites sur des oppositions binaires ${ }^{2}$, principalement: 1) sur les oppositions entre les sens des racines de leurs unités de nomination, qui résument les entités communes du monde ${ }^{3} ; 2$ ) sur les oppositions entre les signifiés 
d'unités qui, elles, symbolisent les entités du monde considérées par l'homo loquens comme des entités universelles: les relations biunivoque et univoques, la vie, le temps, le sexe...; ces unités sont dans les langues les premières pièces de leurs systèmes de communication et de nomination ${ }^{4}$.

Les langues de l'homme ne sont pas des langues de hasard. Les animaux qui n'ont pas conscience du temps - leur mémoire est courte - ont, dans leur vie, par réaction, par hasard, mis en tas un nombre limité de cris immuables qui désignent autant d'expériences globales, figées ${ }^{5}$.

La mémoire longue de l'homme lui a donné la conscience du temps; la capacité d'agir, d'inventer un projet, de planifier son action, de choisir, pour réaliser son projet, tel acte, tel objet... Son premier comportement d'homme a dû être ensembliste, binaire. C'est ce même comportement, observable, qui est le sien encore. C'est le seul comportement qu'il puisse avoir, un comportement déterminé par sa capacité de combinatoire binaire, fille du temps, inhérente à sa nature.

L'homme a construit ses langues, comme il inventait, construisait le monde que ses sens lui rapportaient. Il les a construites avec les ressources immédiates de la combinatoire binaire, la première des combinatoires possibles, la seule alors à lui être accessible, simple et puissante.

De leur construction régulièrement binaire, la langue arabe est un témoin éclatant. Le système syllabique $\Sigma$, de l'arabe ne comprend que les deux seules syllabes \{Consonne-Voyelle\} et \{Consonne-Voyelle-Consonne\}. Or, deux séquences données de syllabes CV et CVC ne peuvent composer deux unités de nomination qui s'opposeraient par la seule opposition d'une consonne et d'une voyelle; ce que montre sommairement la disposition en colonnes ci-dessous des deux séquences différentes possibles:

\section{C.CV}

CV.C VC

dont l'opposition entre $\mathrm{C}$ et $\mathrm{V}$ est double, inévitablement ${ }^{6}$.

C'est ce chiasme

$$
\begin{aligned}
& \text { CV } \\
& \text { VC }
\end{aligned}
$$

qui est à l'origine de l'organisation commune aux langues sémitiques, qui est restée jusqu'à hier l'organisation de la langue arabe $\mathrm{f}^{7}$. Ce chiasme, qui a pour cadre le système syllabique de la langue, entraîne dans son fonctionnement la disjonction de ses consonnes et de ses voyelles. Cette disjonction, dès lors que consonnes et voyelles peuvent être utilisées indépendamment les unes des autres, a créé l'opportunité d'un plan général en rendant possible l'attribution systématique aux unes et aux autres de tâches différentes.

FIGURE 1

Plan de la langue arabe

$$
\Sigma=\{\mathrm{CV}, \mathrm{CVC}\} \rightarrow
$$

$$
\{\mathrm{V}\}=\varnothing
$$


La langue arabe, et comme elle, jadis, chacune des langues sémitiques, a construit son système de nomination sur des arrangements de consonnes, les racines de ses unités de nomination. Ainsi:

(1) $/ \mathrm{kal} \varnothing \mathrm{b} /($ de racine $\sqrt{\mathrm{k}}-\mathrm{l}-\mathrm{b})$ chien

Elle a fait de ses voyelles désinentielles les signifiants de ses fonctionnels primitifs, ses cas, par exemple:

(2) $/ \mathrm{kal} \varnothing \mathrm{b}-\mathrm{u} /$ chien (nominatif)

$/ \mathrm{kal} \varnothing \mathrm{b} /$ était un substantif, déclaré comme tel, dans le cadre de ce plan originel, par sa voyelle zéro, / $\varnothing /$; un substantif déclaré par sa voyelle /a/, signifiant de la modalité d'animéité ${ }^{8}$, comme étant le nom d'un être animé; un substantif, par sa nature, étranger au temps, une res: l'âge de l'animal est ignoré par son nom.

Concurremment, le temps était pris en compte par l'opposition entre:

$$
/ \mathrm{V} / \quad \text { et } \quad \mid \varnothing /
$$

La seule présence de cette voyelle étant suffisante pour impliquer le temps, celleci, l'une des trois voyelles brèves de la langue (/a/, /i/, /u/), a pu être utilisée pour la création d'une nouvelle modalité, la modalité d'agentivité, affine au temps. Ainsi, l'exemple suivant:

(3) / kalab/ rage

nomme un état déclaré par sa deuxième voyelle /a/, l'un des signifiants de la modalité d'agentivité, comme produit par une réaction, ici une affection, c'est-à-dire la conséquence de la morsure d'un chien. Quant à la première voyelle /a/, elle est dans cette nouvelle forme le signifiant d'une autre modalité, la diathèse, qui ne peut exister que dans le temps.

La perception naïve du temps comme une entité continue, indéfinie, monotone, a suscité des bornes à son étendue et des modalités à son déroulement. La relation au temps propre à la langue arabe est signifiée par des modalités aspectuelles. Ces modalités déterminent chaque modus - action, réaction, état - non pas extrinsèquement en le datant par référence à une donnée temporelle, qui serait une autre entité que lui, mais en représentant son déroulement propre, intrinsèquement, par autoréférence.

Un chiasme intervient dans l'expression de chacun des deux couples d'aspects que la langue possède:

- un couple propre aux modus d'action et d'état ${ }^{9}$;

- un couple propre aux verbes, qui sont des modus conjoints à des personnes.

Les deux aspects du verbe arabe s'opposent par la progression achevée ou non de leurs modus vers un terme. L'un, qui peut être dit achevé, montre comme ayant atteint son terme le modus qu'il détermine. Lautre, qui peut être dit non achevé, montre comme n'ayant pas atteint son terme le modus qu'il détermine. 
Chaque verbe arabe est construit sur deux racines: la racine de son modus, faite d'un certain agencement de trois consonnes, $\sqrt{ } \mathrm{CCC}^{10}$, et la racine de la "personne» toujours associée au modus, son sujet ou son objet, faite, quant à elle, d'une seule consonne $\sqrt{ } \mathrm{C}$.

Cette composition du verbe, qui est sa composition minimale, peut être ordonnée. L'ordre $\sqrt{ } \mathrm{CCC}-\sqrt{ } \mathrm{C}$ dit le modus achevé, tandis que l'ordre inverse $\sqrt{\mathrm{C}}-\sqrt{\mathrm{CCC}}$ dit le modus non achevé:

(4) $\sqrt{\text { dearab }}-\sqrt{\text { ta }}$ tu as frappé

(5) $\sqrt{\text { ta }}-\sqrt{\text { diribu }}$ tu frappes, frapperas

Ce chiasme se trouve être aussi iconique, montrant dans l'achevé le modus derrière le sujet ou l'objet, son passé, et, dans le non achevé, le modus devant lui, son présent, son futur ${ }^{11}$. Le modus d'action, d'aspect achevant, a pour schème $\sqrt{\mathrm{Ca}} \mathrm{CiC}$ :

(6) $\sqrt{\text { da:rib }}$ frappant

Le modus d'état, d'aspect non achevant, a pour schème $\sqrt{ } \mathrm{CaCi}: \mathrm{C}$ :

(7) $\sqrt{\text { dari:b }}$ frappeur, brutal

Dans le premier de ces deux chiasmes aspectuels, les racines échangeaient leurs places. Dans le deuxième, ce sont les voyelles longue et brève - l'unité est construite sur une seule racine - qui échangent leurs places.

$$
\begin{aligned}
& \mathrm{V}: \mathrm{V} \\
& \mathrm{VV} \text { : }
\end{aligned}
$$

Ces deux chiasmes sont évidents, lisibles: chacune des séquences est le signifiant immédiat d'un signifié.

Un chiasme différent sert à distinguer, de nouveau par leurs positions, deux modus homophones qui ont pour racine, l'un et l'autre, la même consonne occlusive glottale sourde / $/$. La voyelle /a/, imposée par le patron syllabique, s'est jointe au signifiant de chacun d'eux pour le faire entrer dans le cadre du système syllabique de la langue ${ }^{12}$. Le modus positionné devant le nom est le modus d'appel, qui est une action de $J e^{13}$, par exemple:

(8) / Ra walad - u/

ô enfant!

Le modus positionné derrière le nom est le modus d'exclamation, qui est une réaction de $J e$, par exemple:

(9) $* /$ ?al walad a?/ $>\quad * /$ Ral walad a:/

par transformation de /a?/ en la voyelle longue /a:/; puis, enfin:

(10) $* /$ Ral walad a:/ $>\quad \begin{aligned} & \text { /Ral walad a/ } \\ & \text { L'enfant } !^{14}\end{aligned}$

par abrègement de la longueur de /a:/; la nouvelle voyelle /a/ désormais confondue avec la voyelle brève signifiant du cas accusatif. 
Ainsi, le chiasme a été oblitéré d'abord par le relâchement de l'articulation qui a entraîné dans /a?/ la transformation de / $/$ / en la longueur de /a/, puis par l'abrègement de la voyelle longue, confondue avec la voyelle casuelle homophone.

Un chiasme différent encore se trouve, sans relief, sans utilité, dissimulé, dans les translatifs /ma:/ et $/ \mathrm{Ran} /{ }^{15}$. Ces deux translatifs sont construits sur la même consonne glottale / $\mathrm{P} /$ qui est ici le signifiant du translatif de la langue non spécifié aspectuellement. Dans /ma:/, / $/$ / est, spécifié par le morphème $/ \mathrm{m} /$ qui le connecte à l'achevé. Dans / $\mathrm{Pan} /, / \mathrm{P} /$ est spécifié par le morphème /n/ qui le connecte au non achevé. La voyelle /a/, titulaire du rôle, sépare les consonnes $/ \mathrm{m} /$ et $/ \mathrm{n} /$ de la consonne $/ 2 /$. D'où les cheminements:

$$
\begin{aligned}
& \{/ \mathrm{m} /-/ \mathrm{R} /\}>\quad * / \mathrm{ma} / \mid>\quad / \mathrm{ma} / / \\
& \{/ \mathrm{P} /-/ \mathrm{n} /\}>/ \mathrm{Pan} /
\end{aligned}
$$

illustrés par les exemples suivants :

(11) /bacda ma: d̦araba 1 kalb-a/ après qu'il eut frappé le chien...

(12) /qabla Pan jaḍriba 1 kalb-a/ avant qu'il ne frappe le chien...

Ainsi, les structures de la composition originelle de / $\mathrm{Ran} /(/ \mathrm{R} / \mathrm{-} / \mathrm{n} /)$ et de /ma:/ $(\mathrm{m} / \mathrm{-} / \mathrm{P} /)$ sont en chiasme. Ce chiasme est le reflet de leur opposition aspectuelle. Mais il a été, lui aussi, oblitéré par le relâchement de l'articulation qui a entraîné dans /ma?/ la transformation de / $/$ / en la longueur de /a/.

Les relatifs de temps /lam:a:/ (au moment [achevé / passé] où...) et /hat:a:/ (au moment [non achevé / non passé] où...), se sont également constitués sur un chiasme. Ce chiasme a été effacé par l'évolution de la langue quand les racines d'une seule consonne n'ont plus été reconnues. Étaient construits sur des racines d'une seule consonne:

- parmi ses unités de nomination qui suivent un certain cours, les modus d'appel, d'exclamation...

- parmi ses unités de nomination saisies en elles-mêmes, les personnes, la res générale, le temps général...

Les relatifs /lam:a:/ et /hat:a:/ sont construits sur la res générale, de racine $\sqrt{\mathrm{m}}$, et sur le temps général, de racine $\sqrt{t}_{\mathrm{t}}$. Leur translatif commun est le translatif / $\mathrm{R} / \mathrm{non}$ spécifié aspectuellement. Le temps général est dans /lam:a:/ spécifié par la modalité /1/, rétrospective; dans /hat:a:/, par la modalité /h/, prospective. Le cheminement de la formation de /lam:a:/ peut être retracé ainsi: $\left\{/ \mathrm{l} /, V_{\mathrm{t}}, \sqrt{\mathrm{m}}_{\mathrm{m}} / \mathrm{R} /\right\}>\star /$ latma?/ (où les deux voyelles /a/ sont les deux voyelles syntagmatiques attendues) $>* /$ lam:a?/ (par assimilation régressive de /t/) > /lam:a:/ (par transformation de / $/$ / en la longueur de $/ \mathrm{a} /)$.

La modalité rétrospective /1/ destine /lam:a:/ à l'achevé, par exemple:

(13) /lam :a: daraba $1 \mathrm{kalb}-\mathrm{a} /$ quand il a frappé le chien

Le cheminement de la formation de /hat:a:/ peut être retracé ainsi: $\{/ \mathrm{h} /, / \mathrm{m} /, / \mathrm{t} /$, $/ \mathrm{R} /\}>>^{*} /$ hamta?/ (où les deux voyelles /a/ sont les deux mêmes voyelles syntagma- 
tiques) $>$ */hamta?/ (par pharyngalisation de /h/, provoquée par /a/, nasalisée, postériorisée donc et pharyngale) $>$ /hat:a:/ (par assimilation régressive de /t/).

La modalité prospective /h/ destine /hat:a:/ au non achevé, par exemple:

(14) /hat:a: jaḍiba 1 kalb-a/ jusqu'à ce qu'il frappe le chien

Les racines de ces deux relatifs façonnent ensemble un simulacre de phrase. Dans /lam:a:/, l'ordre $\left(\sqrt{t}_{\mathrm{t}}-\sqrt{\mathrm{m}}_{\mathrm{m}}\right)$, du temps général et de la res générale, est parallèle à l'ordre (modus - personne) dans le verbe à l'achevé. Dans /hat:a:/, l'ordre $(\sqrt{ } \mathrm{m}-\sqrt{\mathrm{t}})$, de la res générale et du temps général, est parallèle à l'ordre (personne - modus) dans le verbe au non achevé.

Le dernier chiasme qui sera ici étudié ne laisse pas de surprendre. Dans les exemples:

(15) $\sqrt{\text { darab }}-\sqrt{\text { ta }}$

tu as frappé

(16) $\sqrt{\text { ta }}-\sqrt{\text { diribu }}$

tu frappes, frapperas

la voyelle entre la deuxième consonne radicale $r$ et la troisième consonne radicale $b$ (dans les exemples [15] et [16]) est le signifiant de la modalité d'agentivité, affine à la modalité d'animéité des noms, deux modalités qui ne sont plus vivantes.

Dans la protolangue arabe, la modalité d'agentivité surdéterminait la diathèse subjective. Ou bien le sujet du modus était sans pouvoir sur lui (agentivité $/ \varnothing /$ ), ou bien il participait à la production du modus, soit par son action spontanée, volontaire (agentivité $/+/$ ), soit par son action provoquée, par réaction (agentivité $/-/)^{16}$.

Il est remarquable que là où cela était possible, les signifiants de l'agentivité aient été disposés en chiasme.

TABLEAU 1

Signifiants de l'agentivité

\begin{tabular}{|c|c|c|}
\hline \multirow{2}{*}{ Agentivité } & \multicolumn{2}{|c|}{ Diathèse subjective } \\
\cline { 2 - 3 } & Verbe achevé & Verbe non achevé \\
\hline $1+/$ & $/ \mathrm{a} /$ & $/ \mathrm{i} /$ \\
$/-/$ & $/ \mathrm{i} /$ & $/ \mathrm{a} /$ \\
$/ \varnothing /$ & $/ \mathrm{u} /$ & $/ \mathrm{u} /$ \\
\hline
\end{tabular}

Ce chiasme non seulement est ici inutile mais il brouille les paradigmes du verbe $^{17}$. L'organisation idéale des signifiants de l'agentivité aurait été l'organisation montrée dans ce deuxième tableau:

TABLEAU 2

Signifiants idéaux de l'agentivité

\begin{tabular}{|c|c|c|}
\hline \multirow{2}{*}{ Agentivité } & \multicolumn{2}{|c|}{ Diathèse subjective } \\
\cline { 2 - 3 } & Verbe achevé & Verbe non achevé \\
\hline$/+/$ & $/ \mathrm{i} /$ & $/ \mathrm{i} /$ \\
$/-/$ & $/ \mathrm{a} /$ & $/ \mathrm{a} /$ \\
$/ \varnothing /$ & $/ \mathrm{u} /$ & $/ \mathrm{u} /$ \\
\hline
\end{tabular}


Dans cette organisation - l'organisation attendue -, chacun des degrés de la modalité aurait été signifié, clairement, univoquement, par un seul signifiant.

Le chiasme surprenant des degrés d'agentivité /+/ et /-/ pourrait être la conséquence outrée d'un pli pris par l'organisation de la langue se construisant.

Il a l'apparence d'un ornement. De fait, si la systématique rigoureuse des langues ne peut faire de place à une rhétorique de figures, elle peut accepter non seulement quelque ornement mais aussi des modalités d'emphase comme dans les modus des exemples suivants:

(17) /j - aḥmu:r/

(homme) qui, par tempérament, parle avec abondance

(18) /t - ašra:b/

fait de boire immodérément

C'est encore une modalité d'emphase que la langue a tenté de se donner en créant une modalité nouvelle d'agentivité dominante, $/++/$ : le sujet marqué par elle l'emporte constamment sur ses opposants, ses rivaux... La langue, pour signifier cette quatrième modalité d'agentivité, a dû inventer un nouveau couple de signifiants.

Ce nouveau couple unit la voyelle /a/, signifiant de l'agentivité «+», et la voyelle $/ \mathrm{u} /$, signifiant de l'agentivité $/ \varnothing /$, ici réemployée pour signifier la constance de cette agentivité.

TABLEAU 3

Signifiants de l'agentivité dominante

\begin{tabular}{|c|c|c|}
\hline \multirow{2}{*}{ Agentivité } & \multicolumn{2}{|c|}{ Diathèse subjective } \\
\cline { 2 - 3 } & Verbe achevé & Verbe non achevé \\
\hline$/++/$ & $/ \mathrm{a} /$ & $\mathrm{u} /$ \\
\hline
\end{tabular}

Ce nouveau couple découpe un nouvel ensemble formé du paradigme achevé I $\mathrm{CaCaCa} /$ normalement couplé au non achevé /jaCCiCu/ (exemples repris [14] et [15] : (/ḍaraba///jaḍribu/)), et du paradigme non achevé /jaCCuCu/ normalement couplé à l'achevé $/ \mathrm{CaCuCa} /$, comme dans les exemples suivants:

(19) /karuma/

il a été généreux

(20) /jakrumu/

il est, sera généreux

Ainsi, la racine $\sqrt{h}_{\mathrm{h}-s ̣-m}$, par exemple, qui dénote la notion de 'querelle' est actualisée au non achevé, non pas dans son paradigme d'agentivité /+/ /jahșịu/ ('il cherche querelle'), mais dans le paradigme d'agentivité $/ \varnothing /$, /jahșumu/, qui connote un état: le triomphe devient l'apanage du sujet querelleur.

C'est l'exemple des grammairiens:

(21) /ha:șama ni:/

il m'a cherché querelle

/fa ha ṣamtu hu Pahș̣mu hu/

et j'ai eu, j'ai le dessus sur lui dans cette querelle 
Ainsi le lien entre les deux signifiants, /a/ et /u/, constitutifs de cette nouvelle modalité, n'est pas établi en langue; il est assuré par la seule mémoire des locuteurs, à l'instar du lien entre les signifiants /a/ et /i/ de l'agentivité / + / et du lien entre les signifiants /i/ et /a/, de l'agentivité /-/. Quand la langue arabe est entrée dans la croyance des Arabes comme étant une langue d'institution divine, la forgerie rhétorique de nouvelles formes a été condamnée. Elles étaient, au demeurant, sans nécessité pour la communication.

Et la langue allait s'engager dans le repli de sa systématique.

\section{Conclusion : rhétorique et discours}

C'est dans le discours que la rhétorique peut trouver ses figures. En langue, elle ne peut trouver une place mesurée que dans les indifférences de son organisation et dans les hasards de la nomination. Ainsi, l'indifférence de la relation biunivoque offre l'occasion toujours disponible d'un effet facile et fort, comme dans la phrase du mystique irakien al-Muhāsibī, (Celui qui demande des comptes à son âme), mort en l'an 243 de l'hégire (857), auteur superbe qui a écrit:

/mutawaq:ic -u-n ru:h-u ka/

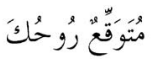

Attente est ton esprit.

(Al-Muhạāibī nc/1978: §15)

et non pas, ce qui aurait été dans l'ordre habituel:

/ru:h-u ka mutawaq:ic ${ }^{\mathrm{c}}-\mathrm{u}-\mathrm{n} /$

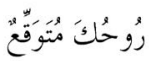

Ton esprit est attente.

Ainsi les hasards de la nomination offrent parfois l'occurrence de deux racines en chiasme, comme dans le vers, de mètre basīt , de Baššār b. Burd, très grand poète irakien, aveugle, l'un des premiers Modernes, mort en l'an 167 ou 168 de l'hégire (784-785):

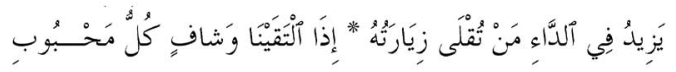

/jazi:du fi: d da:?-i man tuqla: zija:rat-u hu: *

Pida: ltaqaja: wa ša:f-i-n kul:-u maḥbu:b-i-:/

Il ajoute à vos peines, s'il vient à vous rencontrer,

tel dont on fuit la visite. L'être aimé [lui, vous] réconforte.

(Baššār, 1950 1: 196)

où les consonnes radicales qui disent la fuite, $\sqrt{ } \mathrm{q}-1-(j)$, et la rencontre, $\sqrt{ } 1-q-(j)$, sont en chiasme.

Ces quelques cas exceptés, c'est, hors langue (la systématique des langues est une systématique fermée), dans le discours donc (les discours sont ouverts aux choix des locuteurs), que la rhétorique trouve la liberté qui lui est nécessaire, par exemple: 
(25)

- le vers, de mètre kāmil, de ${ }^{\mathrm{c} A n t a r a:}$

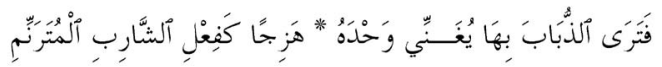

/fa tara: d duba:b-a bi ha: jugan:i: wahd-a hu: *

haziž-a-n ka ficl-i š ša:rib-i l mutaran:im-i:/

$\mathrm{Tu}$ y vois la mouche chanter seule, *

bruissante, pareille au buveur qui fredonne.

('Antara 1390/1970: 197)

- les phrases d'Al-Zahid, reprises de sa Risāla fïl-židd wa l-hazl:

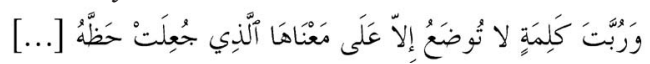

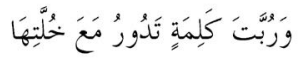

/wa rub:ata kalimat-in la: tu: da ${ }^{c} u$ Pil:a: cala: $m a^{c} n a:$ ha:

l:adi: žucilat had:-a hu [...]

/wa rub:ata kalimat-in tadu:ru maca hul:at-i ha:/

Combien de mots ne s'arrêtent que sur les sens auxquels ils ont été assortis![...]

Et combien de mots vont en ronde avec un partenaire!

(AL-ZAhid 1399/1979: 243)

- le jugement connu qui ricoche sur les grammairiens:

/qawl-u ka Pahwan-u min ḥuža:t-i naḥwij:-i-n/

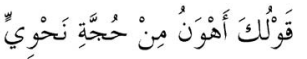

Ce que tu avances est plus inconsistant qu'un argument de grammairien.

\section{NOTES}

1. Voir particulièrement, à l'index, le Traité de rhétorique biblique de R. Meynet.

2. Sur leurs oppositions et non pas sur leurs différences!

3. Ces racines sont les primitives du savoir des hommes.

4. Un signifié est une entité abstraite, un concept, qui apparaît dans un système linguistique comme l'un de ses éléments constitutifs; autrement dit, un signifié est un sens du système.

5. «À la fin des années 1970 [...] Robert Seyfarth et Dorothy Cheney ont montré que des vervets, ou singes verts, dans une réserve du Kenya, avaient trois cris d’alarme différents qui correspondaient aux trois prédateurs susceptibles de les attaquer: le léopard, l'aigle martial et le python [...]» (Picq 2008: 25).

6. En français, différemment, les paires \{aorte vs porte\}, \{poète vs porte\} opposent une voyelle à une consonne; en français, consonnes et voyelles peuvent occuper dans les formes les mêmes positions.

7. En français, le chiasme est particulièrement le signifiant de l'interrogation: Vous venez. par opposition à Venez-vous? Il semble absent de la création lexicale. En témoignent les nombreux néologismes nécessaires impeccablement inventés par André Clas.

8. Les modalités constituent un ensemble complémentaire de l'ensemble des racines. Unités amorphes, elles se greffent sur les racines qu'elles déterminent, qu'elles transforment en unités de nomination fléchies appartenant à des paradigmes.

9. Le modus de réaction n'est plus attesté que sporadiquement; exemple: /ma:raḍ/ (qui tombe malade en conséquence de...).

10. Les racines de quatre consonnes, $\sqrt{ }$ CCCC, sont des racines secondaires, créées par dérivation des formes de la langue à deuxième consonne radicale longue.

11. Observation de Bernard Pottier. 
12. La voyelle /a/ est la voyelle la plus proche de la position de repos de la langue, d'où son choix comme prothèse.

13. L’appel est également réalisé en arabe avec les morphèmes /ja:/ et / Raj:uha:/ ainsi qu'avec les morphèmes, moins fréquents, / Raj:atuha:/, / Raja:/ et / haja:/.

14. /Pal/ est le signifiant correspondant aux articles le, la, les.

15. Les translatifs sont, à côté des coordonnants et des fonctionnels, des pièces du système de communication. C'est par leur moyen qu'une phrase peut être translatée de son statut de phrase (c'està-dire de segment syntaxique maximal et donc indépendant) en un segment dont le statut syntaxique est celui d'une composante de la phrase.

16. Si le modus est objectif, son sujet est de facto d'agentivité nulle.

17. C'est donc le chiasme $\{/ \mathrm{a} /-/ \mathrm{i} /\}$ vs $\{/ \mathrm{i} /-/ \mathrm{a} /\}$ qui a été retenu et non pas le chiasme $\{/ \mathrm{i} /-/ \mathrm{a} /\}$ vs $\{/ \mathrm{a} /$ - /i/\}, également possible. Ces chiasmes sont indifférents au système. Cependant, il a fallu choisir. Le tableau idéal, hypothétique, qui va être présenté, montre dans les voyelles /i/ les signifiants de l'agentivité /+/ et dans les voyelles /a/ les signifiants de l'agentivité /-/. L'affectation des signifiés aux signifiants aurait pu tout aussi bien être inversée.

\section{RÉFÉRENCES}

Al-Muhasibi (nc/1978) : Kitāb at-Tawahhum. André Roman, éd. Paris: Klincksieck.

Al-ŽẠ̣ıD̦ (1399/1979): Rasā’il al-Žahiḍ. 'Abd as-Salām Muhammad HĀrūn, éd. Vol. 1. Le Caire: Maktabat al-Hānžī.

BAššAr ibn Burd (nc/1950-1954-1957-1966) : Dīwān. Muhammad AṬ-TĀHIr b. ' ĀšHūr, éd. 4 vol. Le Caire.

cAntara (1390/1970): Dìwān. Muhammad Sacīd Mawlawī, éd. Beyrouth: al-Maktab al'islāmī.

Meynet Roland (2007): Traité de rhétorique biblique. Paris: Lethellieux.

PicQ, Pascal (2008): Aux sources du langage. In: Pascal PicQ, Laurent SAgart, Ghislaine Dehaene, Cécile Lestienne, dir. La plus belle histoire du langage. Paris: Seuil, 17-74. 